\title{
PRINCIPAL ANALYTIC LINK THEORY IN HOMOLOGY SPHERE LINKS
}

\author{
A. NÉMETHI, WALTER D NEUMANN, AND A. PICHON
}

\begin{abstract}
For the link $M$ of a normal complex surface singularity $(X, 0)$ we ask when a knot $K \subset M$ exists for which the answer to whether $K$ is the link of the zero set of some analytic germ $(X, 0) \rightarrow(\mathbb{C}, 0)$ affects the analytic structure on $(X, 0)$. We show that if $M$ is an integral homology sphere then such a knot exists if and only if $M$ is not one of the Brieskorn homology spheres $M(2,3,5), M(2,3,7), M(2,3,11)$.
\end{abstract}

\section{PRINCIPAL ANALYTIC LINK THEORY}

Let $M$ be a normal surface singularity link. In particular, $M$ is a closed 3-manifold which can be given by a negative definite plumbing.

There may exist many different complex analytic structures on the cone $C(M)$, i.e., many analytically different normal surface singularities $(X, 0)$ whose links $L_{X}$ are homeomorphic to $M$. Our aim is to understand these different analytic structures from the point of view of the "principal analytic link theory" on $M$.

A link or multilink $L=m_{1} K_{1} \cup \ldots \cup m_{r} K_{r} \subset M=L_{X}$ is algebraic if $(M, L)$ is the link $(M, L)=\left(L_{X}, L_{C}\right)$ of a germ pair $(X, C, 0)$ consisting of a normal surface germ and a (not necessarily reduced) complex curve through the singular point $0 \in X$ (this was called "analytic" in [3]). This is a topological property: $L$ is algebraic if the $K_{i}$ are $\mathbb{S}^{1}$-fibres in a negative definite plumbing decomposition of $M$ obtained by possibly applying blow-ups to the minimal negative definite plumbing of $M$.

We say $L$ is principal analytic for $X$ if there exists a holomorphic germ $f:(X, 0) \rightarrow(\mathbb{C}, 0)$ such that the pair $(M, L)$ is homeomorphic to the link $\left(L_{X}, L_{f}\right)$ of the pair $\left(X, f^{-1}(0)\right)$, taking account of multiplicities.

We say that $L=m_{1} K_{1} \cup \ldots \cup m_{r} K_{r} \subset M$ is potentially principal if there exists a normal surface germ $X$ with link $L_{X}=M$ for which $L$ is principal analytic.

According to ([3], Theorem 2.1), the potential principality of an algebraic multilink $L \subset M$ is a topological property which is equivalent to any one of the following 
- The multilink $(M, L)$ is fiberable;

- $[L]=0$ in $H_{1}(M ; \mathbb{Z})$ (note that $[L]$ is always torsion);

- $I^{-1} \mathbf{b}$ is an integral vector, where $I$ is the intersection matrix for the plumbing and $\mathbf{b}$ the vector whose entry corresponding to a plumbing component is the sum of multiplicities of components of $L$ that are fibres of that component.

When $M$ in the link of a rational singularity, then a potentially principal multilink $(M, L)$ is principal analytic for every analytic structure $(X, 0)([1])$. The same conclusion holds when $M$ is the link of a minimally elliptic singularity and $L$ is a knot ([6, Lemma p. 112]).

In [3], we gave several examples of surface singularity links $M$ whose principal analytic link theory is sensitive to the analytic structure in the following sense: for each analytic structure $(X, 0)$ on $C(M)$, there exists a potentially principal knot in $M$ which is not principal analytic for this structure. In fact, we gave examples of pairs of potentially principal links, where the principality of each obstructed the principality of the other.

The aim of this paper is to show that when $M$ is an integral homology sphere ( $\mathbb{Z}$ HS) this behaviour is general, except in the rational and minimally elliptic cases. Our technique consists of constructing a set of principal analytic knots $K_{1}, \ldots, K_{n}$ which are not compatible, i.e., which cannot be realized by germs $f_{i}:(X, 0) \rightarrow(\mathbb{C}, 0)$ from the same analytic structure $(X, 0)$.

Example 1.1. Let $V(p, q, r):=\left\{\left(x_{1}, x_{2}, x_{3}\right) \in \mathbb{C}^{3} \mid x_{1}^{p}+x_{2}^{q}+x_{3}^{r}=0\right\}$ with $p, q, r$ pairwise coprime. Its link $M=M(p, q, r)$ is a $\mathbb{Z}$-homology sphere with three singular fibres $K_{1}, K_{2}, K_{3}$ realized as principal analytic knots by $K_{i}=M \cap\left\{x_{i}=0\right\}$.

Let $K$ be the $(2,1)$-cable on $K_{3} \subset M(2,3,13)$. It is a potentially principal knot in $M=M(2,3,13)$. Let $(Z, p)$ be an analytic structure on the cone $C(M)$ such that $K_{3}$ is realized by a holomorphic function $f_{3}:(Z, p) \rightarrow(\mathbb{C}, 0)$. Then $K$ is not realized by any $f:(Z, p) \rightarrow(\mathbb{C}, 0)$ on $(Z, p)([3], 3,1)$.

Before stating more precisely the main result, let us generalize the notion of principal analytic multilink, and say what we mean by the principal analytic link theory of a surface singularity link $M$.

Definition 1.2. A coloured multilink in $M$ is the data of an algebraic multilink $L \subset M$ with a partition of its components: $L=$ $L_{1} \coprod \ldots \coprod L_{n}$. 
Definition 1.3. A coloured multilink $L=L_{1} \coprod \ldots \coprod L_{n} \subset M$ is principal analytic for a normal surface singularity $(X, 0)$ with link $L_{X}=M$ if there exist analytic germs $f_{i}:(X, 0) \rightarrow(\mathbb{C}, 0), i=1, \ldots, n$ such that

(1) the pair $(M, L)$ is homeomorphic to $\left(L_{X}, L_{f}\right)$, where $f=f_{1} \ldots f_{r}$;

(2) each $\left(M, L_{i}\right)$ is homeomorphic to $\left(L_{X}, L_{f_{i}}\right)$ (note that this does not imply (1) - see Remark 2.5).

We say $L$ is potentially principal if it is principal analytic for some analytic structure $(X, p)$.

Of course, the potential principality of each link $L_{i}$ is a necessary condition for the potential principality of $L$. But it is not sufficient when $n \geq 2$, as shown by the examples of incompatible knots mentioned above: the coloured link $K_{1} \amalg \ldots \coprod K_{n}$ is not potentially principal, but each component is. That is, the knots $K_{1}, \ldots, K_{n}$ can be realized by functions $f_{i}:\left(X_{i}, 0\right) \rightarrow(\mathbb{C}, 0), i=1, \ldots, n$ defined on some analytic structures $\left(X_{i}, 0\right)$ on the cone $C(M)$, but the $\left(X_{i}, 0\right)$ cannot have the same analytical type. So, although the multilink $L=K_{1} \cup \ldots \cup K_{n}$ can be realized by a function $f:(X, 0) \rightarrow(\mathbb{C}, 0)$ for some $(X, 0)$, there is no $(X, 0)$ and $f$ such that $f$ splits into a product $f=f_{1} \ldots f_{n}$ with $f_{i}:(X, 0) \rightarrow(\mathbb{C}, 0)$ realizing the knot $K_{i}$.

Given $M$, let us denote by $\operatorname{PPL}(M)$ the set of potentially principal coloured multilinks $L$ in $M$; we call $\operatorname{PPL}(M)$ the principal analytic theory on $M$. Given a normal surface singularity $(X, 0)$ with link $M$, we denote by $\operatorname{PAL}(X) \subset \operatorname{PPL}(M)$ the set of coloured links $L$ in $M$ which are principally analytic for $(X, 0)$. So $\operatorname{PPL}(M)=\bigcup_{L_{X} \cong M} \operatorname{PAL}(X)$.

The study of the principal analytic link theory on $M$ consists of the two following natural questions:

(1) Describe the set $\operatorname{PPL}(M)$;

(2) describe the subsets $\operatorname{PAL}(X)$ for $(X, 0)$ realizing $M$.

The unique rational singularity with $\mathbb{Z H S}$ link is $(V(2,3,5), 0)$ with link $M(2,3,5)$. There are only two $\mathbb{Z}$ HS links which belong to minimally elliptic singularities: $M(2,3,7)$ and $M(2,3,11)$. Our main result, which is a first important step in this program, is as follows:

Theorem 1.4. Let $M$ be a $\mathbb{Z} H S$ singularity link which is not homeomorphic to $M(2,3,5), M(2,3,7)$ or $M(2,3,11)$. Then there exists an algebraic coloured link $L=K_{1} \coprod \ldots \coprod K_{n}$ which is not in $\operatorname{PPL}(M)$ and such that:

(1) Each $K_{i}$ is a potentially principal knot

(2) $\forall i \neq j,\left(M, K_{i}\right)$ is not homeomorphic to $\left(M, K_{j}\right)$. 
(Of course, since $M$ is a $\mathbb{Z}$-homology sphere, the potential principality of $K_{i}$ is automatic.)

\section{Two CONSTRUCTIONS OF NON-PPL COLOURED LINKS}

In this section, we present through examples two methods (Methods 1 and 2) to construct some coloured links $L$ in a given $M$ such that $L \notin \operatorname{PPL}(M)$ but each $L_{i}$ is in $\operatorname{PPL}(M)$. The first one, which was introduced in [3], could be used in any $M$, whereas the second is only available in a $\mathbb{Z} H S$.

Method 1 (Using the delta invariant of a reduced curve). Let $K$ be a fibred knot in $M$, and let $\Phi: M \backslash K \rightarrow \mathbb{S}^{1}$ be an open-book fibration with binding $K$. We set

$$
\mu(K)=1-\chi\left(\Phi^{-1}(t)\right),
$$

where $t \in \mathbb{S}^{1}$ and where $\chi$ denotes the Euler characteristic. Notice that $\mu(K)$ does not depend on the choice of $\Phi$, and that it can be computed from any plumbing graph of $(M, K)$ (or any splice diagram if $M$ is a QHS).

Let $K_{1} \coprod \cdots \coprod K_{n}, n \geq 2$ be a coloured link whose components $K_{i}$ are potentially principal knots with multiplicity 1 . For each $i=$ $1, \ldots, n-1$, let $\Phi_{i}: M \backslash K_{i} \rightarrow \mathbb{S}^{1}$ be a fibration of $K_{i}$. We consider the coloured multilink $L=K_{1} \coprod \ldots \coprod K_{n-1}$ and we define the semigroup $\Gamma\left(L, K_{n}\right)$ as the semigroup generated by the degrees of the maps $\Phi_{i}$ on the knot $K_{n}$. Notice that these degrees do not depend on the $\Phi_{i}$ 's and can be computed from any plumbing graph of $\left(M, K_{1} \amalg \ldots \amalg K_{n}\right)$. We denote by $\delta\left(L, K_{n}\right)$ the number of gaps in $\Gamma\left(L, K_{n}\right)$, i.e., the number of positive integers that are not in $\Gamma\left(L, K_{n}\right)$.

Lemma 2.1. If $L \coprod K_{n} \in \operatorname{PPL}(M)$ then

$$
\mu\left(K_{n}\right) \leq 2 \delta\left(L, K_{n}\right) .
$$

Proof. Let $(X, 0)$ be such $L \coprod K_{n} \in \operatorname{PAL}(X)$ and let $f_{j}:(X, 0) \rightarrow$ $(\mathbb{C}, 0)$ be a holomorphic germ with link $K_{j}$ for $j=1, \ldots, n$. Then $\mu\left(K_{n}\right)=\mu\left(f_{n}\right)$, the Milnor number of $f_{n}$. According to [2], one has $\mu\left(f_{n}\right)=2 \delta\left(f_{n}\right)$, where $\delta\left(f_{n}\right)$ denotes the $\delta$-invariant of the curve $f_{n}^{-1}(0)$. Recall that $\delta\left(f_{n}\right)$ counts the number of gaps in the semigroup $\Gamma\left(f_{n}\right)$ generated by the all the multiplicities of the holomorphic germs $g:(X, 0) \rightarrow(\mathbb{C}, 0)$ along the curve $f_{n}^{-1}(0)$. Moreover, if $g$ is such a germ then this multiplicity is the degree of the Milnor fibration $g /|g|$ restricted to the link of $f_{n}^{-1}(0)$. Since $K_{1}, \ldots, K_{n-1}$ can be realized by germs $f_{1}, \ldots, f_{n-1}$, we have $\Gamma\left(L, K_{n}\right) \subset \Gamma\left(f_{n}\right)$, so $\delta\left(f_{n}\right) \leq \delta\left(L, K_{n}\right)$. 
Example 2.2 (Non-PPL coloured link). Let $M$ be the link of the Brieskorn-Pham singularity $z_{1}^{3}+z_{2}^{4}+z_{3}^{5}=0$ and and let $K_{i}, i=1,2,3$ be the end-knots in $M$ corresponding to $z_{i}=0$. Let us consider the $(2,5)$-cabling $K$ on the link $K_{3}$ of $z_{3}=0$. Its splice diagram is as follows.

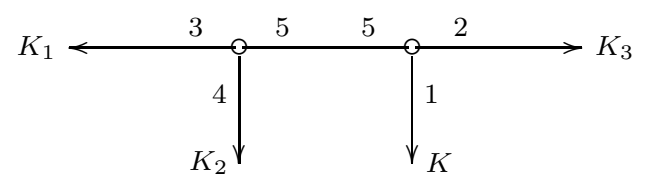

The semigroup $\Gamma\left(K_{1} \coprod K_{2} \coprod K, K_{3}\right)$, being generated by 3,4 and 5 , has two missing numbers, whereas $\mu\left(K_{3}\right)=(3-1)(4-1)=6>4$.

Thus, by Lemma 2.1, the coloured link $L=K_{1} \coprod K_{2} \coprod K_{3} \coprod K$ is not realized on any $(X, 0)$, i.e., $L \notin \operatorname{PPL}(M)$

Method 2 (Using the semigroup condition). Method 2 is based on the so-called End-Curve Theorem for $\mathbb{Z H S}$ links:

Theorem 2.3 ([5], theorem 4.1). Let $(X, 0)$ be a normal surface singularity with $\mathbb{Z} H S$ link $M$. Let $\Delta$ be a splice diagram of $M$ such that $\Delta$ is the minimal splice diagram of the pair $(M, L)$, where $L$ denotes a link whose components are the end-knots of $\Delta$.

Assume that for each of the end leaves of $\Delta$, there exits a function $z_{i}:(X, 0) \rightarrow(\mathbb{C}, 0)$ whose link is the corresponding end-knot. Then:

(1) The graph $\Delta$ satisfies the semigroup condition;

(2) $X$ is a complete intersection of embedding dimension $\leq n$;

(3) the functions $z_{1}, \ldots, z_{n}$ generate the maximal ideal of the local ring $\mathcal{O}_{(X, 0)}$, and $X$ is a complete intersection of splice type with respect to these generators.

This result furnishes an alternative argument to prove that the $L$ of the previous example does not belong to $\operatorname{PPL}(M(3,4,5))$. Indeed, if $L \in \operatorname{PAL}(X)$ for some analytic structure $(X, 0)$ on $C(M)$ then each leaf of the splice diagram of the figure is realized by a function $(X, 0) \rightarrow(\mathbb{C}, 0)$, so, by the End-Curve Theorem, $(X, 0)$ is splice. But the semigroup condition is not realized at the right hand node, as $5 \notin\langle 3,4\rangle$.

More generally, if a splice diagram does not satisfy the semigroup condition, the coloured link consisting of all end-knots for the diagram is not in $\mathrm{PPL}(M)$ by the End-Curve Theorem, so Theorem 1.4 is proved in this case.

Not all splice diagrams for $\mathbb{Z H S}$ singularity links satisfy the semigroup conditions. Nevertheless, Method 2 gives a short proof of a weak version of Theorem 1.4. 
Theorem 2.4 (Weak Version of Theorem 1.4). Let $M$ be a $\mathbb{Z}$-homology sphere which is the link of a normal surface singularity. If $M$ is not homeomorphic to $M(2,3,5)$ then there exists a coloured link $L=$ $K_{1} \coprod \ldots \coprod K_{n} \notin \operatorname{PPL}(M)$, consisting of knots $K_{i} \in \operatorname{PPL}(M)$.

Proof. Let $G$ be the minimal splice diagram of $M$. Let us consider an end-node of $G$ as in the figure below, with $a_{1}<\ldots<a_{n}$. Let $K_{1}, \ldots, K_{n}$ be end-knots as marked, and $K_{n+1}, \ldots, K_{r}$ the end-knots corresponding to the remaining leaves (lying in the portion $G^{\prime}$ ).

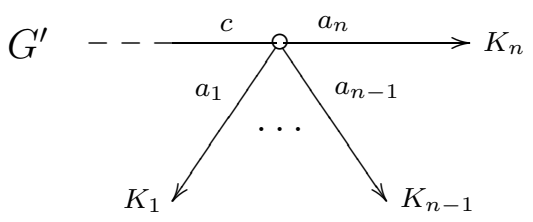

We can assume $c>1$, since we have already proved the result if the semigroup condition fails. Denote

$$
A=\prod_{i=1}^{n-1} a_{i} ; \quad A_{j}=A / a_{j}, j=1, \ldots, n-1 .
$$

Set $\alpha=c A_{n-1}$. Assume we can choose $d \in\{\alpha+1, \alpha+2\}$ such that $d \notin\langle\alpha, A\rangle$. Replace $K_{n}$ by two parallel $(1, d)$ cablings on $K_{n}$ as shown.

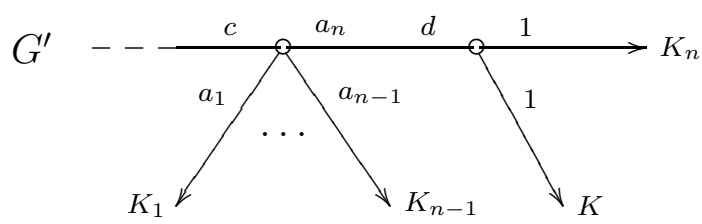

As $a_{n} d>\alpha a_{n-1}$, the splice diagram of $\left(M, K_{n} \cup K\right)$ satisfies the edge determinant condition. However, the semigroup condition is not realized at the right hand node as $d<c A_{j}$ for $j<n-1$ and $d \notin$ $\langle\alpha, A\rangle$. Thus, by the End-Curve Theorem, the fibered coloured link $L=K_{1} \coprod \ldots \coprod K_{r} \coprod K$ does not belong to $\operatorname{PPL}(M)$. This completes the proof, assuming that $d$ exists.

If $d$ above does not exist then $n=2$ and $\left(c, a_{1}\right)=(2,3)$ or $(c, 2)$ with $c$ odd. In the latter case, if $c \geq 7$ then $d=c-2$ satisfies $a_{2} d>a_{1} c$ but fails the semigroup condition, so we may assume $c=3$ or 5 .

Moreover, if $G^{\prime}$ does not just consist of a single vertex, then the smallest multiple of $a_{1}$ that can contribute to the semigroup is $2 a_{1}$ so we see that the semigroup condition still fails with $d=3$ if $\left(c, a_{1}\right)=(2,3)$ and with $d=c+2$ if $\left(c, a_{1}\right)=(3,2)$ or $(5,2)$. Finally, for the one-node diagram $G$ with weights $2,5, a_{2}$ we can use $d=3$ while for $2,3, a_{2}$ we 
can use $d=1$ (recall $a_{2} \geq 7$ in both cases since we ruled out $M(2,3,5)$ ).

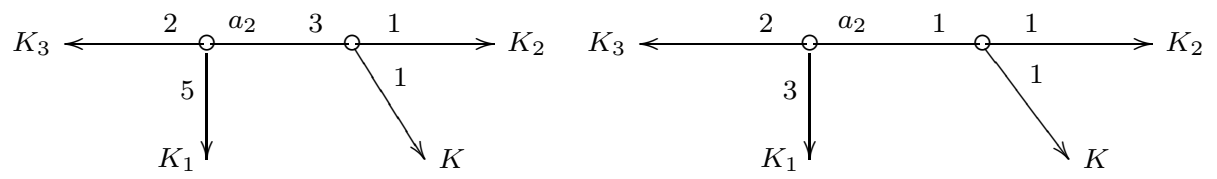

Remark 2.5. The knots $K_{n}$ and $K$ are isotopic. That is why Theorem 2.4 is a weak version of Theorem 1.4. The positions of isotopic knots with respect to each other can make a big difference. In the following two splice diagrams (with $a \geq 7$ ) the knots $K_{2}, K_{2}^{\prime}, K_{2}^{\prime \prime}$ and $K_{2}^{\prime \prime \prime}$ are mutually isotopic.

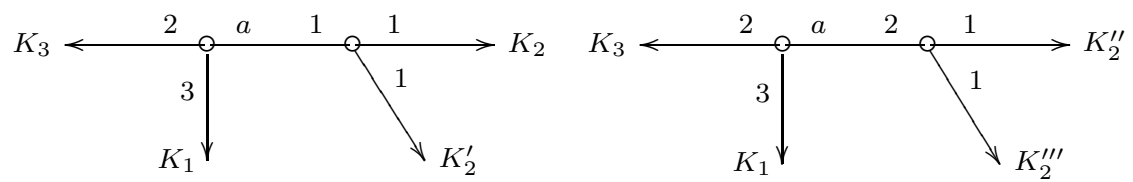

As just proved, the left colored link is not in $\operatorname{PPL}(M)$. But for $a=7$ or 11 the right one is in $\operatorname{PAL}(X)$ for every analytic structure $X$ on the cone $C(M)$. The reason is that $X$ is minimally elliptic and $K_{2}^{\prime \prime}$ and $K_{2}^{\prime \prime \prime}$ are realized by generic hyperplane sections.

\section{Proof of Theorem 1.4}

3.1. The case of Brieskorn-Pham links. Let $M$ be the link of the Brieskorn-Pham singularity $z_{1}^{p}+z_{2}^{q}+z_{3}^{r}=0$ where $p<q<r$ are pairwise coprime integers. Let $K_{i}, i=1,2,3$ be the end-knots in $M$ corresponding to $z_{i}=0$.

If there exists $s \in \mathbb{N}$ such that

$$
r s>2 p q \text { and } s \notin\langle p, q\rangle \quad(*),
$$

then, using the semigroup condition (Method 2), one obtains that the four-coloured link $L=K_{1} \coprod K_{2} \coprod K_{3} \coprod K$ does not belong to $\operatorname{PPL}(M)$, where $K$ denotes the $(2, s)$-cabling on $K_{3}$ :

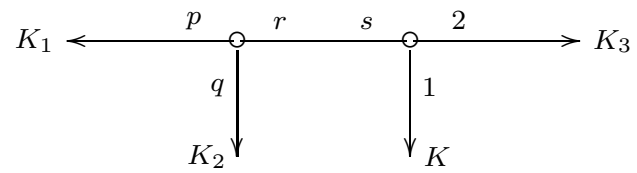

First, assume that $p>2$. The integers in the semigroup $\langle p, q\rangle$ which are $\leq 2 p+1$ belong to $\{p, q, 2 p, p+q\}$. Then if $q \notin\{p+1,2 p+1\}$, $s=2 p+1$ satisfies condition $(*)$.

If $q=p+1$, the integers in the semigroup $\langle p, q\rangle$ which are $\leq 2 p+3$ belong to: $\{p, p+1,2 p, 2 p+1,2 p+2,3 p\}$. Then, if $p \neq 3, s=2 p+3$ 
satisfies condition $(*)$ as $2 p+2<2 p+3<3 p$, and if $(p, q)=(3,4)$, then one can choose $s=5$ as in the example of section 2 .

If $q=2 p+1$ and $p>3$, then $s=2 p+3$ satisfies condition $(*)$, and if $(p, q)=(3,7)$, one can take $s=11$.

It remains to deal with the case that $p=2$. If $q>5$, then $s=5$ satisfies condition $(*)$. If $(p, q)=(2,5)$, then $r \geq 7$ and $s=3$ satisfies $(*)$. If $(p, q)=(2,3)$, then $r \geq 13$ (as we avoid the rational case $r=5$ and minimally elliptic cases $r=7,11)$ and $s=1$ satisfies $(*)$.

This completes the proof for Brieskorn-Pham links.

3.2. The case of a Seifert link. Assume that the 3 -manifold $M$ is Seifert, or equivalently that the minimal splice diagram $G$ of $M$ has a single node. Let $n$ be the number of incident leaves. We assume that $n \geq 4$, as the case $n \leq 3$, which corresponds to the Brieskorn-Pham case, has already been treated. Let $a_{1}, \ldots, a_{n}$ be their weights, indexed in such a way that $a_{1}<\ldots<a_{n}$, and let $K_{1}, \ldots, K_{n}$ be corresponding end-knots. We set:

$$
A=\prod_{i=1}^{n-1} a_{i} ; \quad A_{j}=A / a_{j}, j=1, \ldots, n-1 .
$$

We argue as in the Brieskorn-Pham case: If there exists $s \in \mathbb{N}$ such that

$$
a_{n} s>2 A \text { and } s \notin\left\langle A_{1}, \ldots, A_{n-1}\right\rangle \quad\left(*_{2}\right),
$$

then the $(n+1)$-coloured link $L=K_{1} \coprod \ldots \coprod K_{n} \coprod K$ does not belong to $\operatorname{PPL}(M)$, where $K$ denotes the $(2, s)$ - cabling on $K_{n}$ (see figure).

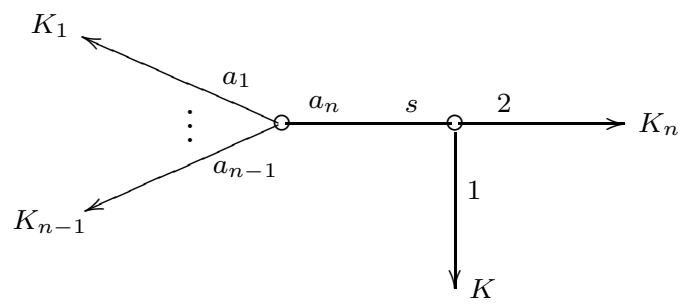

We will show that $s=2 A_{n-1}+1$ satisfies $\left(*_{2}\right)$.

First notice that $s$ satisfies the inequality of condition $\left(*_{2}\right)$. Moreover, as $n \geq 4$, we have

$$
6 \leq A_{n-1}<A_{n-2}<\ldots<A_{1}
$$

and for each $i \in\{2, \ldots, n-1\}$, one has $A_{i-1}-A_{i} \geq 3$. Thus the integers in the semigroup $\left\langle A_{j} ; j=1, \ldots, n-1\right\rangle$ which are $\leq 2 A_{n-1}+1$ must be among $A_{n-1}, \ldots, A_{1}$ and $2 A_{n-1}$, and hence divisible by one of the $a_{i}$ 's with $i<n-1$. So $2 A_{n-1}+1$ cannot be in this semigroup, so it satisfies $\left(*_{2}\right)$. 
Remark. One can also prove Theorem 1.4 in the case of a Seifert link using only Method 1, with much more complicated cases. The advantage of such a proof is that Method 1 can be used in any 3dimensional manifold with the same underlying splice diagram, even those having genus at the nodes and leaves, whereas the second method is specific to $\mathbb{Z} H S$ 's. So one obtains an extension of Theorem 1.4 to a larger family of normal singularity links.

The idea of the proof in the case of a Brieskorn-Pham link $M=$ $\mathbb{S}_{\epsilon}^{3} \cap\left\{z_{1}^{p}+z_{2}^{q}+z_{3}^{r}=0\right\}$ is the following: using again the notation $K_{1}$, $K_{2}, K_{3}$ for the end-links, the generic cases are treated by performing a string of cablings on $K_{3}$ giving rise to $x+1$ knots $K_{0}^{\prime}, \ldots, K_{x}^{\prime}$ as in the figure below, where $x=2 a+1$.

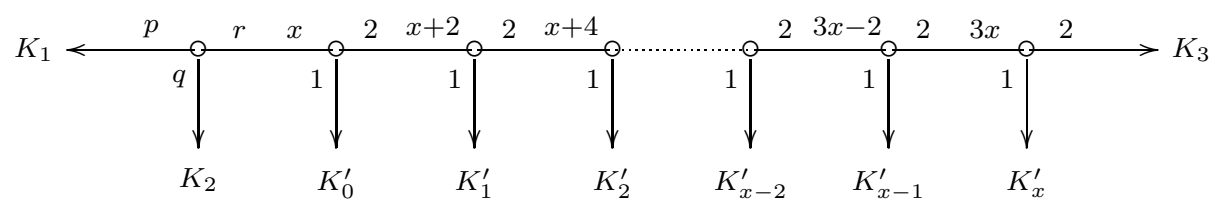

Then we show that, except for a finite numbers of particular cases, we have the inequality:

$$
\mu\left(K_{3}\right)>\delta\left(\mathcal{L}, K_{3}\right)
$$

where $\mathcal{L}=\left\{K_{1}, K_{2}, K_{0}^{\prime}, \ldots, K_{x}^{\prime}\right\}$. So, by Lemma 1 , the $(\mathrm{x}+4)$-coloured link

$$
K_{1} \coprod K_{2} \coprod K_{3} \coprod K_{0}^{\prime} \coprod \ldots \coprod K_{x}^{\prime}
$$

does not belong to $\operatorname{PPL}(M)$.

The particular cases which cannot be treated by these cablings, but which can be solved independently by hand are the following: $(p, q) \in\{(2,3),(2,5),(2,7),(3,4),(3,5),(4,5),(4,7),(6,7)\}$. The details are left to the reader. The proof in the general Seifert case is a generalization of this one. It is likely that a similar proof exists for a general normal surface singularity with $\mathbb{Z} H S$ link, but we have not attempted it.

3.3. Proof of Theorem $\mathbf{1 . 4}$ in the non-Seifert case. Let us assume that the splice diagram $G$ has at least two nodes. Choose an end-node $(\nu)$ of the splice diagram $G$, that is, a node which is an end-vertex of the diagram obtained by removing all leaves from $G$ (so it has at most a single incident edge which is not a leaf).

3.3.1. First case: $(\nu)$ has 4 or more incident edges. Let $n+1$ be the number of incident edges of $\nu$. Denote by $a_{1}<\ldots<a_{n}$ the weights on the adjacent leaves and by $r$ that on the remaining adjacent edge. 
(We can assume $r>1$ if we want, since otherwise the diagram fails the semigroup condition, and we have already proved this case.)

Let $K_{1}, \ldots, K_{n}, K_{n+1}, \ldots, K_{m}$ be end-knots corresponding to all the leaves of $G$, the knots $K_{1}, \ldots, K_{n}$ corresponding to the leaves adjacent to $\nu$. We set

$$
A:=\prod_{i<n} a_{i} ; \quad A_{j}:=\prod_{i \neq j, n} a_{i}, \text { for } j=1, \ldots, n-1 .
$$

We argue as before: If there exists $s \in \mathbb{N}$ such that

$$
a_{n} s>2 r A_{n-1} a_{n-1} \text { and } s \notin\left\langle A, r A_{1}, r A_{2}, \ldots, r A_{n-1}\right\rangle \quad\left(*_{3}\right),
$$

then the $(m+1)$-coloured link $L=K_{1} \coprod \ldots \coprod K_{m} \coprod K$ does not belong to $\operatorname{PPL}(M)$, where $K$ denotes the $(2, s)$-cabling on $K_{n}$.

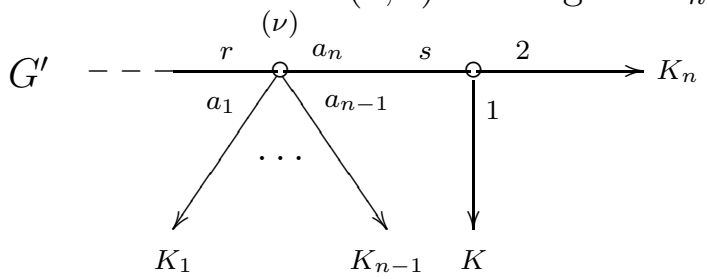

We will show that $s=2 r A_{n-1}+1$ satisfies $\left(*_{3}\right)$.

As $a_{n}>a_{n-1}, s=2 r A_{n-1}+1$ satisfies the inequality of condition $\left(*_{3}\right)$. We again have

$$
6 \leq A_{n-1}<A_{n-2}<\ldots<A_{1}
$$

and $A_{i-1}-A_{i} \geq 3$ for $2 \leq i \leq n-1$.

Since $r A_{i}>r A_{n-1}+3 r$ for $i<n-1$, the integers in the semigroup $\Gamma=\left\langle A, r A_{1}, r A_{2}, \ldots, r A_{n-1}\right\rangle$ which are $\leq 2 r A_{n-1}+1$ must be among

$$
2 r A_{n-1}, n A, r A_{i}+n A, \quad n=0,1,2, \ldots
$$

These are all divisible by some $a_{i}$ with $i<n-1$ and $2 r A_{n-1}+1$ is not, so it cannot be in the semigroup.

3.3.2. Second case: $(\nu)$ has 3 incident edges. We denote by $p<q$ the weights of the two adjacent leaves and by $r$ that of the remaining edge.

If $r<q$, then we can use the same argument as in the BrieskornPham case. (In fact the argument is simplified by the fact that the semigroup in the argument is now smaller than $\langle r, p\rangle$ since it is contained in the semigroup generated by $r, 2 p, 3 p, \ldots$. So some cases of the earlier argument are not needed and a $(2,2 t+1)$-cabling at the $q$-weighted leaf works with $t=\min (p, r)$ unless $r=2 p+1$, in which case $(2,2 p+3)$-cabling works.)

Let us now assume $p<q<r$. Let $K_{1}$ and $K_{2}$ be end-knots corresponding to the leaves weighted by $p$ and $q$ respectively, and let 
$K_{3}, \ldots, K_{m}$ be end-knots corresponding to the other leaves of the splice diagram $G$.

For each $i=3, \ldots, m$, let $\alpha_{i}^{\prime}$ be the product of the weights adjacent to the path joining the leaf $K_{2}$ to the leaf $K_{i}$. As $p$ divides $\alpha_{i}^{\prime}$, we set $\alpha_{i}^{\prime}=p \alpha_{i}$, where $\alpha_{i} \geq 2$. If there exists $s \in \mathbb{N}$ such that

$$
q s>2 p r \text { and } s \notin\left\langle r, \alpha_{3} p, \ldots, p \alpha_{m}\right\rangle \quad\left(*_{4}\right),
$$

then the $(m+1)$-coloured link $L=K_{1} \coprod K_{2} \coprod K_{3} \coprod \ldots \coprod K_{m} \coprod K$ does not belong to $\operatorname{PPL}(M)$, where $K$ is the $(2, s)$-cabling on $K_{2}$ :

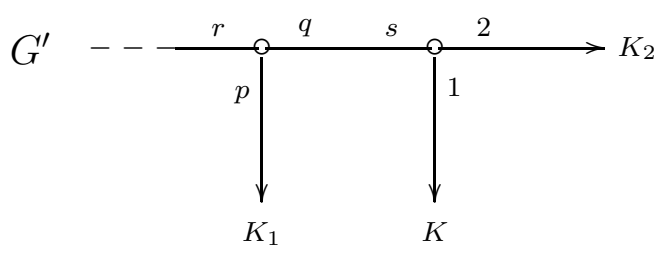

As $p<q, s=2 r+1$ and $s=2 r+3$ both satisfy the inequality of condition $\left(*_{4}\right)$. Assume that $2 r+1$ and $2 r+3$ both belong to the semigroup $\left\langle r, \alpha_{3} p, \ldots, p \alpha_{m}\right\rangle$. There then exist $\kappa, \gamma \in\left\langle\alpha_{3}, \ldots, \alpha_{m}\right\rangle$ such that

$$
\begin{array}{lll}
2 r+1=\kappa p & \text { or } \quad 2 r+1=r+\kappa p, & \text { and } \\
2 r+3=\gamma p & \text { or } \quad 2 r+3=r+\gamma p .
\end{array}
$$

(The possibilities $2 r+3=2 r+\gamma p$ and $2 r+3=3 r$ are ruled out by the facts $\gamma>1$ and $r \geq 5$.) Thus there are four possible cases:

Case 1. $2 r+1=\kappa p$ and $2 r+3=\gamma p$. Then $2=(\gamma-\kappa) p$, so $p=2$, so $2 r+1=2 \kappa$. Contradiction.

Case 2. $2 r+1=r+\kappa p$ and $2 r+3=\gamma p$, which leads to $1=(\gamma-2 \kappa) p$. Contradiction.

Case 3. $2 r+1=\kappa p$ and $2 r+3=r+\gamma p$, which leads to $5=(2 \gamma-\kappa) p$. Therefore $p=5$. As $2 \leq p<q<r, s=2 r-1$ satisfies the inequality of condition $\left(*_{4}\right)$. Assume that $2 r-1$ and $2 r+1$ both belong to $\left\langle r, 5 \alpha_{3}, \ldots, 5 \alpha_{m}\right\rangle$. There then exist $\lambda, \delta \in\left\langle\alpha_{3}, \ldots, \alpha_{m}\right\rangle$ such that

$$
\begin{aligned}
& 2 r+1=5 \lambda \text { or } 2 r+1=r+5 \lambda, \quad \text { and } \\
& 2 r-1=5 \delta \quad \text { or } \quad 2 r-1=r+5 \delta \text {. }
\end{aligned}
$$

This leads to four possible cases:

(1) $2 r+1=5 \lambda$ and $2 r-1=5 \delta$. Then $2=5(\lambda-\delta)$. Contradiction.

(2) $2 r+1=r+5 \lambda$ and $2 r-1=5 \delta$, which leads to $3=5(2 \lambda-\delta)$. Contradiction.

(3) $2 r+1=r+5 \lambda$ and $2 r-1=r+5 \delta$, which leads to $2=5(\lambda-\delta)$. Contradiction 
(4) $2 r+1=5 \lambda$ and $2 r-1=r+5 \delta$, which leads to $3=5(\lambda-2 \delta)$. Contradiction

Case 4. $2 r+1=r+\kappa p$ and $2 r+3=r+\gamma p$. This leads to $2=(\gamma-\kappa) p$, so $p=2$. If $q \geq 5$ then $s=r+2$ satisfies condition $\left(*_{4}\right)$. So we may assume $q=3$. Then $r \geq 5$ and for $r=5$ we can take $s=7$, so we may assume $r \geq 7$.

Since we have dealt with all other possibilities, we can assume now that every end-node of $G$ has this form, i.e., it is valence 3 with two leaves with weights 2 and 3 and the third edge having weight $\geq 7$.

Let $(\nu)$ now be an end-node of the graph obtained by deleting the 2 - and 3-weighted leaves at the end-nodes of $G$. So the picture is as follows:

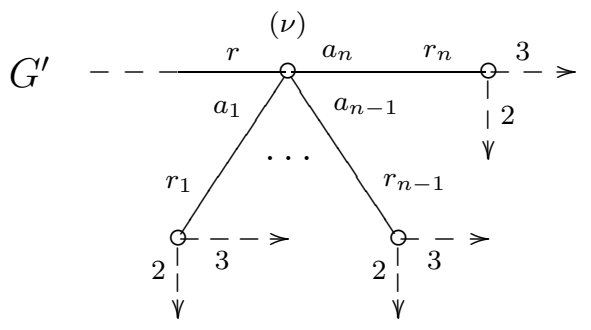

The dashed pairs of $(2,3)$-weighted leaves may or may not exist (but at least one pair must exist; note that the weight $r_{i}$ is $\geq 7$ but is omitted if the $(2,3)$-pair of leaves does not exist at that vertex). We assume $a_{1}<\cdots<a_{n}$, and denote, as usual, $A=a_{1} \ldots a_{n-1}$ and $A_{j}=A / a_{j}$ for $j=1, \ldots, n-1$.

We first consider the case that there is no $(2,3)$-pair at the end of the $a_{n}$-weighted edge:

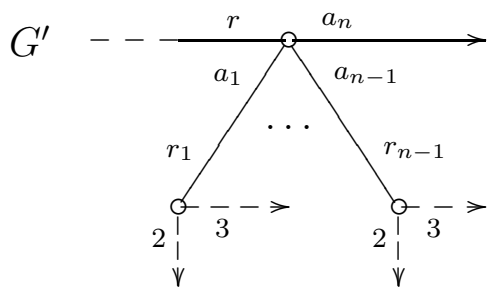

Then the same arguments as before reduce us to the case that $n=2$, $a_{1}=2, a_{2}=3$, and then the following cabling does not satisfy the semigroup condition and thus resolves this case:

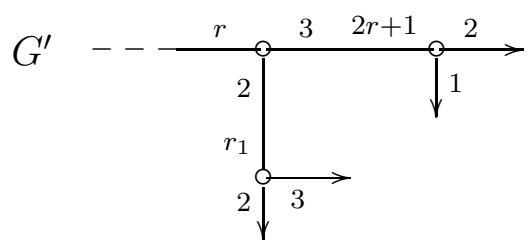


Indeed, the relevant semigroup is generated by $2 r, 3 r$, and a subset of $2 \mathbb{N}$, so it does not contain $2 r+1$.

We may now assume there is a $(2,3)$-pair at the $a_{n}$-weighted edge. If there is an integer $k$ with $\frac{r_{n}}{6}>k>\frac{r A}{a_{n}}$ then the following internal cabling gives an admissible splice diagram for $M$, which fails the semigroup condition since $1 \notin\langle 2,3\rangle$ :

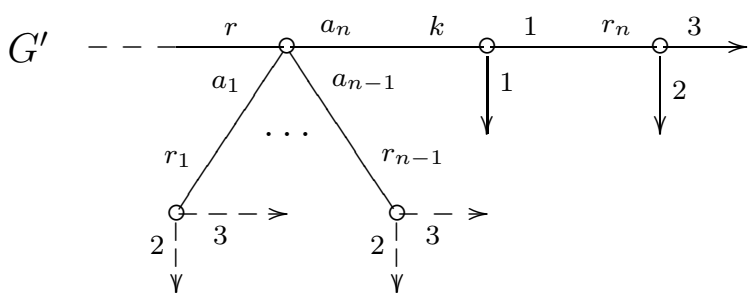

So we may assume that there is no integer $k$ with $\frac{r_{n}}{6}>k>\frac{r A}{a_{n}}$ (but $\left.\frac{r_{n}}{6}>\frac{r A}{a_{n}}\right)$. In particular, $r A_{n-1} \geq\left\lceil\frac{r_{n}}{6}\right\rceil$, since $r A_{n-1}$ is an integer larger than $\frac{r A}{a_{n}}$. We thus have:

$$
r A_{1}>\cdots>r A_{n-1} \geq\left\lceil\frac{r_{n}}{6}\right\rceil .
$$

Consider the cabling:

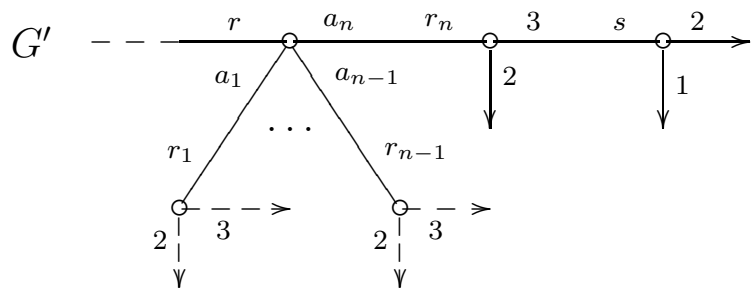

in which we need $4 r_{n}<3 s$ to have an admissible diagram. Let $s=$ $r_{n}+2 x$ with $x=\left\lceil\frac{r_{n}}{6}\right\rceil+\epsilon, \epsilon \in\{0,1\}$. Then $3 s=3 r_{n}+6 x>4 r_{n}$, as desired. Moreover, since $r_{n}>6$, we have $x<r_{n}$.

Since $s=r_{n}+2 x$ is odd and $x<r_{n}$, if $s$ satisfies the semigroup condition then $x$ must be in the semigroup generated by $r A_{1}, \ldots, r A_{n-1}$ and $A$. Thus if both $x=\left\lceil\frac{r_{n}}{6}\right\rceil$ and $x=\left\lceil\frac{r_{n}}{6}\right\rceil+1$ are in this semigroup there are just two possibilities:

$$
\begin{aligned}
\left\lceil\frac{r_{n}}{6}\right\rceil & =r A_{n-1} \quad \text { and } A \text { divides }\left\lceil\frac{r_{n}}{6}\right\rceil+1, \quad \text { or } \\
\left\lceil\frac{r_{n}}{6}\right\rceil+1 & =r A_{n-1} \text { and } A \text { divides }\left\lceil\frac{r_{n}}{6}\right\rceil .
\end{aligned}
$$

Both cases imply $n=2$, since otherwise $a_{1}$ is a divisor of both $\left\lceil\frac{r_{n}}{6}\right\rceil$ and $\left\lceil\frac{r_{n}}{6}\right\rceil+1$. Moreover, they imply there is no $(2,3)$-pair at the end of the $a_{1}$-edge, since if there were then $r A_{n-1}=r$ would not be available in the semigroup. 
Now suppose that $x=\left\lceil\frac{r_{n}}{6}\right\rceil+2$ is also in the semigroup. In the first case $x=r+2$; the only possibilities are $r=2$ (so $a_{1}=3$ since $A=a_{1}$ divides $r+1$ ) or $a_{1}=2$. In either case we need $a_{1}$ to be in the semigroup, so the $r$-weighted edge is a leaf, so node $(\nu)$ is an end-node, so the weights of its leaves are 2 and 3 and $a_{2} \geq 7$. The second case similarly implies that node $(\nu)$ must be an end-node so its pair of leaves is $(2,3)$-weighted and the third weight $a_{2}$ is $\geq 7$.

Since $a_{2} \geq 7$ the inequalities $\frac{r_{n}}{6}>k>\frac{r \bar{A}}{a_{n}}\left(=\frac{6}{a_{2}}\right)$ are satisfied by $k=1$, so we are in a case which we had already dealt with, and the proof is complete.

\section{REFERENCES}

[1] M. Artin, On isolated rational singularities of surfaces. Amer. J. Math. 88 $1966129-136$.

[2] R.-O. Buchweitz and G.-M. Greuel, The Milnor number and deformations of complex curve singularities. Invent. Math. 58 (1980), 241-281.

[3] W.D. Neumann and A. Pichon, Complex analytic realization of links. Intelligence of low dimensional topology 2006, 231-238, Ser. Knots Everything, 40, World Sci. Publ., Hackensack, NJ, 2007.

[4] W.D. Neumann and J. Wahl, Complete intersection singularities of splice type as universal abelian covers, Geometry and Topology, Vol. 9 (2005), 699-755.

[5] W.D. Neumann and J. Wahl, Complex surface singularities with integral homology sphere links, Geometry and Topology, Vol. 9 (2005), 757-811.

[6] M. Reid, Chapters on Algebraic Surfaces, Complex algebraic geometry (Park City UT, 1993), IAS/Park City Math. Ser. 3, Amer. Math. Soc. (Providence, RI, 1997), 3-159. 